\title{
A mouth rinse based on a tea solution of Salvia officinalis for oral discomfort in palliative cancer care: a randomized controlled trial
}

\author{
Ragnhild Elisabeth Monsen ${ }^{1,2}$ (1) Bente Brokstad Herlofson ${ }^{3,4} \cdot$ Caryl Gay $^{5,6} \cdot$ Katrine Gahre Fjeld $^{7}$. \\ Lene Hystad Hove $^{7} \cdot$ Karl Egil Malterud $^{8} \cdot$ Elisabeth Saghaug $^{1} \cdot$ Joran Slaaen $^{1} \cdot$ Tone Sundal $^{1} \cdot$ Anita Tollisen $^{1}$. \\ Anners Lerdal ${ }^{2,5}$
}

Received: 28 May 2020 / Accepted: 21 January 2021 / Published online: 14 February 2021

(C) The Author(s) 2021

\begin{abstract}
Background Few clinical studies evaluate interventions to reduce oral discomfort among patients in palliative care. Aim This study examines the efficacy of a Salvia officinalis (SO) based herbal mouth rinse compared to conventional normal saline (NS) in order to improve oral health.

Design A block-randomized controlled trial. Data were collected before and after a 4-day intervention with either SO ( $n=44)$ or NS ( $n=44)$. Numerical rating scales (NRS, 0-10) and 12 items from the European Organisation for Research and Treatment of Cancer (EORTC) Quality of Life Questionnaire-Oral Health 17 (EORTC QLQ-OH17) measured patient-reported oral symptoms. An oral examination was performed before and after the intervention.

Setting/participants This study included adult patients with late-stage cancer in an inpatient hospice unit.

Results Of the 88 patients included (mean age $=63.9$ years, $\mathrm{SD}=10.6), 73(83 \%)$ completed the study. At baseline, $78 \%$ reported dry mouth on the EORTC QLQ-OH17, and $80 \%$ rated dry mouth $\geq 4$ on the NRS. Total oral health scores based on the 12 EORTC QLQ-OH17 items improved similarly in both groups $(p<0.001)$. However, dry mouth ratings on both the EORTC QLQ$\mathrm{OH} 17$ ( $p=0.036)$ and NRS $(p=0.045)$ improved more in the SO group than in the NS group. Plaque on the teeth improved in both the SO $(p=0.008)$ and NS $(p=0.018)$ groups, but plaque on the tongue and erythema only improved with NS.

Conclusions This study did not detect an overall significant difference between SO and NS. Both mouth rinses improved oral health parameters, indicating that systematic assessment and oral care may reduce oral discomfort.
\end{abstract}

Trial registration NCT02067572

Keywords Palliative care $\cdot$ Oral health $\cdot$ Oral care $\cdot$ Mouth rinse $\cdot$ Salvia officinalis $\cdot$ Randomized controlled trial

\section{Introduction}

Oral discomfort is highly prevalent in palliative care patients. Moreover, oral symptoms are not limited to patients with head

Ragnhild Elisabeth Monsen ragnhm@uio.no

1 Department of Medicine, Lovisenberg Diaconal Hospital, Oslo, Norway

2 Department for Interdisciplinary Health Sciences, Institute of Health and Society, Faculty of Medicine, University of Oslo, Postboks 1089 Blindern, 0317 Oslo, Norway

3 Department of Oral Surgery and Oral Medicine, Faculty of Dentistry, University of Oslo, Oslo, Norway and neck cancer, but are rather common for patients with all types of cancer [1]. Despite its prevalence, poor oral health among cancer patients, particularly those receiving palliative care, is underreported by patients and health staff [2] and is a

$4 \quad$ Unit of Oral and Maxillofacial Surgery, Department of Otorhinolaryngology - Head and Neck Surgery Division for Head, Neck and Reconstructive Surgery, Oslo University Hospital, Oslo, Norway

5 Department of Research, Lovisenberg Diaconal Hospital, Oslo, Norway

6 Department of Family Health Care Nursing, University of California, San Francisco, CA, USA

7 Department of Cariology and Gerodontology, Faculty of Dentistry, University of Oslo, Oslo, Norway

8 Department of Pharmacy, Section Pharmaceutical Chemistry, University of Oslo, Oslo, Norway 
neglected aspect of patient care [1]. Given that a primary goal of palliative care is to relieve pain and distressing symptoms, research aimed at addressing patients' oral comfort is needed.

Side effects from cancer and/or cancer treatment often affect patients' oral health and may cause oral complications, such as xerostomia (subjective experience of dry mouth), salivary gland hypofunction (low saliva secretion), oral infection, dysphagia, mucositis, denture associated stomatitis, oral ulceration, caries, coated mouth and tongue, orofacial pain or dysgeusia (altered taste) [2-5]. Xerostomia is particularly common, with a prevalence of approximately $80 \%$ among palliative cancer care patients in Norway, while other common oral symptoms, such as plaque on teeth and tongue, infection, oral ulceration, and pain, have a prevalence of 30 $50 \%[1,6]$. These symptoms may cause nutritional problems, weight loss, and fatigue and can negatively impact social interaction. Saliva lubricates and moistens the oral mucosa, facilitates speech, eating, and swallowing, and plays a significant role in maintaining oral health. Thus, common oral symptoms and discomfort have significant negative impacts on patients' quality of life [4, 7-10].

Several mouth rinses and products currently exist to treat or manage common oral symptoms, particularly xerostomia [11-14], but there is insufficient evidence to support their long-term or relative effectiveness [15-17]. There is also a multitude of mouth rinses for preventing caries, treating infections, and reducing inflammation [18]. However, a lack of knowledge among nurses and physicians of these products and their effectiveness for treating common oral symptoms may lead to inappropriate guidance and oral hygiene practices.

Moreover, differences in the severity of side effects associated with cancer and its medical treatment may require individual approaches when choosing products. For example, low saliva production can affect taste and sensitivity to the texture of products [9]. This may result in patients preferring a product solely on the basis of its taste or application method, rather than its effectiveness. Thus, an optimal solution for patients would be to introduce a variety of suitable oral health care mouth rinses that are both comfortable to use and proven effective to reduce distressing oral symptoms.

The Norwegian guidelines for palliative care recommend normal saline (NS) $(0.9 \%$ sodium chloride) rinse as a saliva substitute [6]. However, sage, Salvia officinalis L. (SO), has a long history in alternative medicine and has been used to treat dyspepsia, pharyngitis, stomatitis, and inflammation in the mouth or throat [19-21]. It has also been suggested to be beneficial for oral discomfort given its anti-inflammatory, antiseptic, antibacterial, antifungal, and metabolic regulation properties [22-24], and this use has been indicated by the European Medicines Agency [23].

For many years, SO has been used in our hospice unit and reported by our patients to be a pleasant and preferred alternative to NS for relieving oral discomfort. However, SO's subjective and clinical effects have not been evaluated in a randomized controlled trial (RCT). Therefore, the aim of this study was to investigate the effect of a SO-based herbal mouth rinse compared with NS mouth rinse on perceptions of oral discomfort and symptoms in a RCT among patients receiving palliative care.

\section{Methods}

This study was designed as a prospective, single-blinded, block-randomized controlled trial to evaluate the effect of SO-based mouth rinse on the oral health of patients with advanced cancer in palliative care compared to the current standard of care [6]. It is a sub-study of the project Oral Health in Advanced Cancer (OralHAC) [25]. The overall aim of the OralHAC project is to improve the care of patients with latestage cancer by addressing their oral health and improving symptom management of oral discomfort. In the current study, data were collected at Lovisenberg Diaconal Hospital in Oslo, Norway, from February 2014 to September 2016. The Regional Committee for Medical Research Ethics, Health Region South-East Norway approved the study in October 2013 (reference \#2013/1531). The study was registered with ClinicalTrials.gov (reference \#NCT02067572) in February 2014. Randomization was performed from a web-based randomization service in three blocks of 20 subjects and a final block of 28 subjects. The allocation sequence was concealed from the researchers enrolling participants by sequential numbered, opaque sealed envelopes. An analysis was conducted after two blocks $(n=40)$ to determine whether there was sufficient beneficial effect from the SO rinse to continue the study.

\section{Sample}

Patients were recruited from a 12-bed inpatient hospice unit, where they were being treated with palliative care. Study inclusion criteria were as follows: (1) admission to the Hospice Lovisenberg inpatient unit, (2) $\geq 18$ years of age and able to provide written informed consent, (3) diagnosis of advanced cancer, and (4) patient report of current oral discomfort. Patients were excluded if they (1) had an estimated life expectancy of $\leq 2$ weeks, (2) currently used antifungal medication, (3) were currently receiving head or neck radiation therapy, (4) were epileptic, (5) had a diagnosis of diabetes, or (6) had any condition that would have interfered with study participation (e.g., inability to use a mouth rinse, anxiety about dental examination or treatment). Exclusion criteria (4) and (5) are related to potential negative interaction with the SO, such as epileptiform convulsion and hypoglycemic activity [26]. Informed written consent was obtained from all study participants. 
Of the 538 patients admitted to the hospice unit during the study period, 514 (96\%) were evaluated for eligibility (Fig. 1).

\section{Variables and measures}

The patients' demographic and medical characteristics were obtained from their medical records. In order to describe the study sample, Karnofsky Performance Status (KPS) was used as a measure of functional performance, with scores ranging from 0 (death) to 100 (perfect health) [27]. Patients reported on their current oral care, including their frequency of tooth brushing and types of equipment used, like soft toothbrush, toothpaste, mouth rinse, floss, interdental brush, toothpick, and tongue cleaner.

The primary outcome for this study was the patientreported outcome of oral symptoms, which were assessed on study days 1 and 5 to obtain measures before and after the 4day intervention. Outcome data were collected by a nurse and a dentist who were blinded to patients' treatment conditions and the mouth rinse used. The patient-reported outcome was assessed by using 12 items (\#31-\#42) selected from the European Organisation for Research and Treatment of Cancer (EORTC) Quality of Life Questionnaire-Oral Health 17 Phase III version (EORTC QLQ-OH17) [28], which is a supplementary module to the core questionnaire (EORTC QLQ-C30) [29]. At the time data collection was planned, the last version of the EORTC QLQ-OH15 Phase IV was not yet available [30]. The EORTC QLQ-OH17 measures oral problems and symptoms during the past week using a 4-point scale (1=not at all, 2=a little, 3=quite a bit, and 4=very much). Because the intervention lasted only 4 days, the timeframe was modified from "during the past week" to "during the past

Fig. 1 Participant flow chart

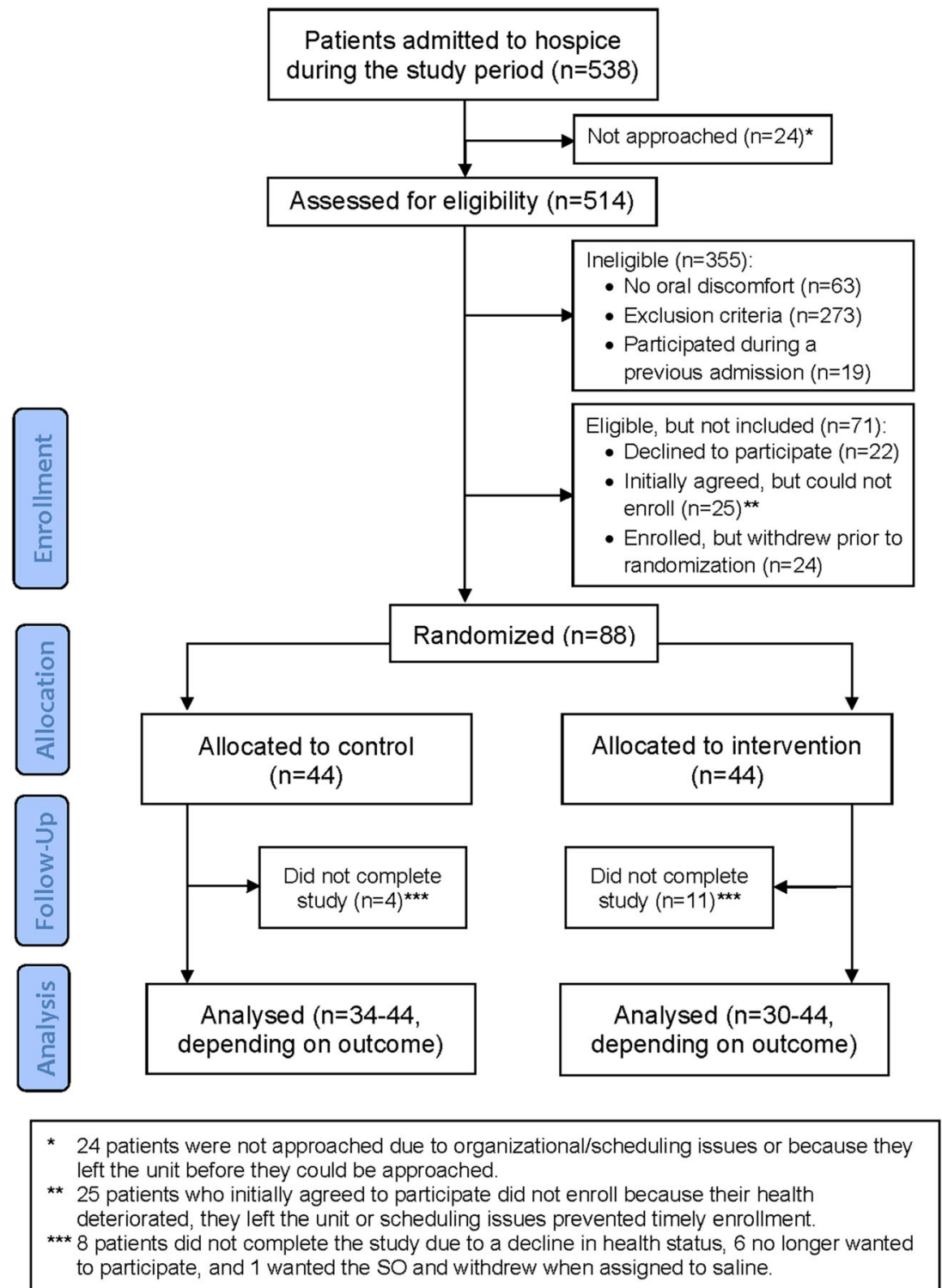


day." Due to the challenges of conducting research in palliative care and to reduce participant burden, we also decided to use only the most relevant questions from the EORTC QLQOH17 module as single symptom items.

In addition, the patients' daily experiences of (1) oral pain, (2) mouth dryness, (3) difficulty swallowing, and (4) pain while swallowing were assessed using a numerical rating scale (NRS) ranging from 0 (no discomfort) to 10 (maximum discomfort). Scores 1-3 indicate mild discomfort, 4-6 moderate discomfort, and 7-10 severe discomfort. NRS items were assessed between 12:00 and 14:00 each study day. On day 5 , upon intervention completion, patients also answered the question: "What do you think about the mouth rinse?" using a NRS ranging from 0 (pleasant experience) to 10 (unpleasant experience). The use of the daily NRS allowed for the assessment of patients' day-to-day experiences of the most common and/or distressing oral symptoms.

The secondary outcome was an objective assessment of oral health based on a clinical examination performed by a dentist before and after the intervention (days 1 and 5).

The oral examination included the dentist's assessment of oral dryness, plaque on the teeth and tongue, the oral mucosa, the total number of teeth, and use of dentures and root remnants. Clinical evaluation of oral dryness was assessed by the sliding mirror test, drawing a dental mirror along the buccal mucosa, scored no/yes. When the mirror slid easily along the mucosa with no friction, it indicated no oral dryness, while friction indicated oral dryness [31]. Plaque on teeth was scored by using the plaque score index of the MucosalPlaque Score (MPS) assessment tool published by Henriksen and coworkers [32]. It rates the plaque from 1 to 4 ( $1=$ no easily visible plaque, $2=$ small amounts of hardly visible plaque, $3=$ moderate amounts of plaque, and $4=$ abundant amounts of confluent plaque). Plaque on tongue was scored using the same rating 1-4. Oral mucosal inflammation was scored by Oral Mucositis Assessment Scale (OMAS) [33], a detailed assessment tool for signs of mucositis: ulceration and erythema. Ulceration was scored $0-3$, where $0=$ no lesion, $1=$ $<1 \mathrm{~cm}^{2}, 2=1-3 \mathrm{~cm}^{2}$, and $3=>3 \mathrm{~cm}^{2}$. Erythema was scored 0 2 , where $0=$ none, $1=$ mild, and $2=$ severe.

\section{Procedure and intervention}

Both the intervention and control groups followed the same standardized procedure, which included basic oral care with tooth brushing morning and evening, rinsing with the assigned solution $(10-15 \mathrm{ml})$ twice for $30 \mathrm{~s}$ four times a day, and after each rinsing, oral gel (1 $\mathrm{cm}$ ) and lip balm were applied. In all patients, the same oral care products were used, such as a soft toothbrush (Jordan ${ }^{\circledR}$ Gum Protector), toothpaste (Biotene $\left.{ }^{\circledR}\right)$, oral gel (Biotene $\left.{ }^{\circledR}\right)$, and lip balm (Biotene $\left({ }^{\circledR}\right)$. The procedures were carried out four times daily, except for tooth brushing, which was performed every morning and evening. Patients could use the assigned rinse as needed in between the four daily intervention procedures.

The control group rinsed with NS and the intervention group with a SO solution consisting of $2.5 \mathrm{~g}$ SO herbal tea/100 $\mathrm{ml}$ water. The SO herbal tea solution was based on dry extract of Salvia officinalis leaves from the Hospital Pharmacy (Sanivo Pharma AS), which were steeped for $2 \mathrm{~min}$ in boiled water. The mouth rinses were prepared and replaced every morning during the intervention period and stored at room temperature, available for the patient as needed. Each rinsing (including extra rinses) was noted in the patient's study journal. When required, nursing staff assisted patients with the rinsing procedure.

\section{Data analysis}

Data were analyzed using SPSS 24.0 for Windows (IBM Corp, Armonk, NY). Descriptive statistics summarized demographic, medical, and mouth care variables. Continuous variables were presented as means and standard deviations (SD), and categorical variables as numbers and percentages. Chisquare tests and Fisher's exact tests were used to compare groups on categorical variables. McNemar's test was used to evaluate within-group change on categorical variables. Independent sample $t$ tests were used to compare groups on continuous variables at specific timepoints, and paired $t$ tests were used to evaluate within-group change on continuous variables. Linear mixed models were used to evaluate group differences over time, as indicated by significant group-byday interactions. Analyses were conducted based on intention-to-treat. $p$ values $<0.05$ were considered statistically significant. Cohen's $d \geq 0.40$ was considered a clinically significant effect size [34].

A power analysis indicated that a sample size of 88 (44 in each group) was sufficient to detect group differences over time (interaction) with a moderate effect size of $f=0.25$, assuming correlations of $r=0.35$ between repeated measures [35], a significance level of 5\% and statistical power of $80 \%$.

\section{Results}

\section{Sample characteristics}

Sample characteristics for the 88 enrolled patients are summarized in Table 1. There were no significant group differences at baseline, except that use of opiate medication was more frequent in the intervention group than the control group. There was no significant group difference on any measure of dental status (Table 1). At baseline, nearly all patients (90\%) reported brushing their teeth twice or more daily, $41 \%$ used 
Table 1 Sample demographic and clinical characteristics

\begin{tabular}{|c|c|c|c|c|}
\hline & $\begin{array}{l}\text { Total sample } \\
(n=88)\end{array}$ & $\begin{array}{l}\text { Control } \\
(n=44)\end{array}$ & $\begin{array}{l}\text { Intervention } \\
(n=44)\end{array}$ & $p$ value \\
\hline Age, years & & & & 0.728 \\
\hline Mean (SD) & $63.9(10.6)$ & $63.5(11.8)$ & $64.3(9.4)$ & \\
\hline Range & $29-84$ & $29-84$ & $45-83$ & \\
\hline Sex, \% (n) & & & & 0.151 \\
\hline Male & $27 \%(24)$ & $20 \%(9)$ & $34 \%(15)$ & \\
\hline Female & $73 \%(64)$ & $80 \%(35)$ & $66 \%(29)$ & \\
\hline Smoker & $(n=83)$ & $(n=42)$ & $(n=41)$ & 0.748 \\
\hline Yes, \% (n) & $23 \%(19)$ & $21 \%(9)$ & $24 \%(10)$ & \\
\hline BMI & $(n=76)$ & $(n=38)$ & $(n=38)$ & 0.811 \\
\hline Mean (SD) & $22.2(3.8)$ & $22.3(4.1)$ & $22.1(3.6)$ & \\
\hline Range & $13.6-32.2$ & $16.4-32.2$ & $13.6-28.2$ & \\
\hline Karnofsky score & $(n=83)$ & $(n=43)$ & $(n=40)$ & 0.504 \\
\hline Mean (SD) & $52.1(16.9)$ & $53.3(18.4)$ & $50.8(17.2)$ & \\
\hline Range & $20-80$ & $20-80$ & $20-80$ & \\
\hline Primary diagnosis, \% (n) & & & & $0.325^{\mathrm{a}}$ \\
\hline Gastrointestinal cancer & $26 \%(23)$ & $29 \%$ & $23 \%(10)$ & \\
\hline Lung cancer & $17 \%(15)$ & $16 \%(7)$ & $18 \%(8)$ & \\
\hline Gynecologic cancer & $16 \%(14)$ & $23 \%(10)$ & $9 \%(4)$ & \\
\hline Prostate cancer & $3 \%(3)$ & $5 \%(2)$ & $2 \%(1)$ & \\
\hline Breast cancer & $13 \%(11)$ & $9 \%(4)$ & $16 \%(7)$ & \\
\hline Other cancer & $25 \%(22)$ & $18 \%(8)$ & $32 \%(14)$ & \\
\hline Head/neck & $8 \%(7)$ & $7 \%(3)$ & $9 \%(4)$ & \\
\hline Metastases & $(n=87)$ & $(n=44)$ & $(n=43)$ & \\
\hline Yes, \% $(n)$ & $83 \%(72)$ & $82 \%(36)$ & $84 \%(36)$ & 0.814 \\
\hline Number of medications & $(n=85)$ & $(n=44)$ & $(n=41)$ & 0.161 \\
\hline Mean (SD) & $11.4(4.2)$ & $10.8(4.6)$ & $12.1(3.6)$ & \\
\hline Range & $4-26$ & $4-26$ & $4-20$ & \\
\hline Type of medical treatment, $\%$ (n) & $(n=85)$ & $(n=44)$ & $(n=41)$ & \\
\hline Steroids & $58 \%(49)$ & $50 \%(22)$ & $66 \%(27)$ & 0.188 \\
\hline Opiates & $85 \%(72)$ & $77 \%(34)$ & $93 \%(38)$ & 0.049 \\
\hline Anti-depressants & $24 \%(20)$ & $20 \%(9)$ & $27 \%(11)$ & 0.489 \\
\hline Blood pressure medication & $21 \%(18)$ & $16 \%(7)$ & $27 \%(11)$ & 0.218 \\
\hline Paracetamol & $59 \%(50)$ & $57 \%(25)$ & $61 \%(25)$ & 0.697 \\
\hline Cardiac medication & $22 \%(19)$ & $25 \%(11)$ & $20 \%(8)$ & 0.544 \\
\hline Bisphosphonate therapy & $7 \%(6)$ & $5 \%(2)$ & $10 \%(4)$ & 0.427 \\
\hline \multicolumn{5}{|l|}{ Cancer treatment, $\%(\mathrm{n})$} \\
\hline Previous radiation therapy & $55 \%(47)$ & $57 \%(25)$ & $52 \%(22)$ & 0.679 \\
\hline on head/neck & $16 \%(14)$ & $18 \%(8)$ & $14 \%(6)$ & 0.560 \\
\hline Previous chemotherapy & $86 \%(74)$ & $84 \%(37)$ & $88 \%(37)$ & 0.592 \\
\hline Current chemotherapy & $33 \%(28)$ & $36 \%(16)$ & $29 \%(12)$ & 0.487 \\
\hline Dental status, mean (SD) & $(n=84-86)$ & $(n=43-44)$ & $(n=41-42)$ & \\
\hline Teeth count & $23.6(6.8)$ & $23.8(6.7)$ & $23.4(6.9)$ & 0.803 \\
\hline Dentures count & $0.4(1.5)$ & $0.3(0.7)$ & $0.5(2.1)$ & 0.144 \\
\hline Root remnant count & $0.2(0.6)$ & $0.1(0.3)$ & $0.3(0.8)$ & 0.142 \\
\hline
\end{tabular}

${ }^{\mathrm{a}}$ Fisher's exact test; ${ }^{\mathrm{b}} n=80$ for total sample, $n=43$ for control, and $n=37$ for intervention toothpicks and 29\% used a mouth rinse. Of the 88 enrolled patients, 15 (17\%) dropped out before completing the study, 4 in the control group, and 11 in the intervention group $(p=0.087)$ (Fig. 1). 


\section{Baseline symptoms of oral discomfort}

Of the 12 oral symptoms assessed using items from the EORTC QLQ-OH17, the most common and severe symptom was dry mouth, with $78 \%$ of patients experiencing oral dryness either "quite a bit" or "very much" (Fig. 2). Other common symptoms of oral discomfort included problems enjoying meals, food and drink tasting different, and sticky saliva, with $>70 \%$ of patients reporting these symptoms at least "a little".

On baseline NRS ratings of oral discomfort, $94 \%$ of patients reported some dry mouth (rating $>0$ ) and $80 \%$ reported moderate to severe dry mouth (rating $\geq 4$ ), with no difference between groups $(p=0.872)$. About half of the patients $(48 \%)$ reported some difficulty swallowing and $24 \%$ reported moderate to severe difficulty swallowing, again with no significant difference between groups $(p=0.109)$. Because $<20 \%$ of patients reported any pain in their mouth or when swallowing, and $<8 \%$ rated them $\geq 4$ on the NRS, these symptoms were not included in subsequent analyses.

Baseline clinical evaluations indicated that most patients had at least small amounts of hardly visible plaque on both the tongue (67\%) and teeth (58\%). Fewer than 10\% had abundant amounts of confluent dental plaque. In addition, at baseline, $47 \%$ of patients had a positive sliding mirror test indicating oral dryness. On the baseline OMAS scoring, $76 \%$ of patients had at least some evidence of erythema (score $>0$ ), but only $5 \%$ had scores $>1$. With respect to ulceration, $45 \%$ had no evidence of ulceration, and only $4 \%$ had scores $>0.5$.

\section{Evaluation of intervention effects}

The normal saline and Salvia officinalis rinses resulted in similarly improved oral comfort mean scores on the 12 items from the EORTC QLQ-OH17 between study days 1 and 5, ( $p=0.001$ and $p=0.003$, respectively) (Table 2 ). With respect to individual symptoms, both the NS and $\mathrm{SO}$ rinses resulted in improved ratings of dry mouth on EORTC QLQ-OH17 item \#37 and the NRS between days 1 and 5, but the reported improvements were significantly higher in the SO group than in the NS group, as evidenced by the significant group-by-day interaction (Fig. 3).

Clinical evaluation showed significantly improved OMAS erythema scores between days 1 and 5 in the NS group only (Table 3). Significantly reduced plaque on teeth was registered both in the NS and SO groups between days 1 and 5. For plaque on the tongue, both groups showed similar improvement in scores, but only the NS group reached statistical significance. There was no significant difference in patients' experience of the mouth rinses between the NS (mean 3.45, SD 2.86) and SO groups (mean 2.73, SD 2.76, $p=0.72$ ), with higher scores indicating less pleasant experiences.

\section{Discussion}

The present study investigated the effect of a mouth rinse based on the herb Salvia officinalis (SO) compared with standard care using normal saline (NS) as a mouth rinse among hospice patients with late-stage cancer. Our findings showed significant improvement in both groups on patient-reported oral symptoms and on clinical evaluation measures after 4 days of intervention. There were no overall statistically significant group differences between the $\mathrm{SO}$ and saline mouth rinses. Given the palliative care setting, the intervention timeframe was 4 days, which may have limited the observed effects.
Fig. 2 Patient-reported oral symptoms at baseline (12 items from the EORTC QLQ-OH17)

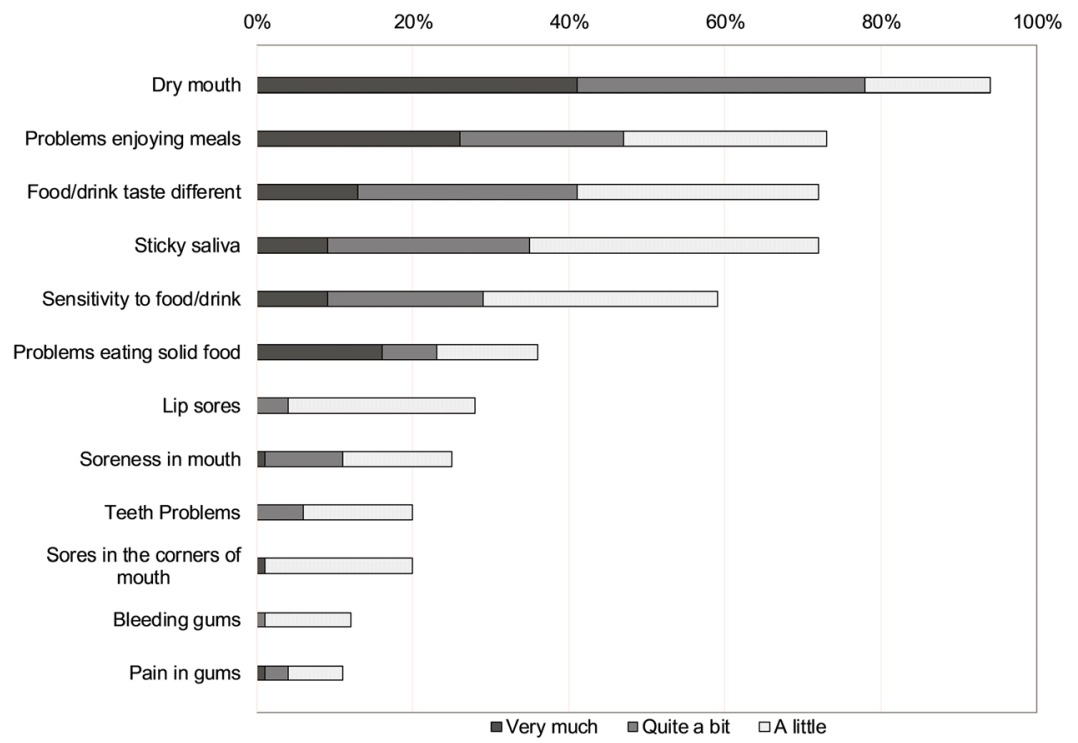


Table 2 Change in patient-reported outcomes between days 1 and 5 by treatment group

\begin{tabular}{|c|c|c|c|c|}
\hline & $\begin{array}{l}\text { Control } \\
(n=44)\end{array}$ & $\begin{array}{l}\text { Intervention } \\
(n=44)\end{array}$ & $\begin{array}{l}p \text { value ( } d \text { value) } \\
\text { for group } t \text { test }\end{array}$ & $\begin{array}{l}p \text { values for mixed } \\
\text { model effects }\end{array}$ \\
\hline \multicolumn{5}{|c|}{ EORTC QLQ-OH17 (1-4 scale) } \\
\hline \multicolumn{4}{|l|}{ 12-item mean score } & \multirow[t]{4}{*}{ GxD p $=0.889 ;$ day $p<\mathbf{0 . 0 0 1}$} \\
\hline Day 1 & $1.80(0.37)(n=43)$ & $1.72(0.39)(n=40)$ & $p=0.305$ & \\
\hline Day 5 & $1.62(0.31)(n=39)$ & $1.50(0.40)(n=33)$ & $p=0.149$ & \\
\hline Day paired $t$ test & $p=\mathbf{0 . 0 0 1}(n=39 ; d=0.58)$ & $p=\mathbf{0 . 0 0 3}(n=33, d=0.56)$ & & \\
\hline \multicolumn{4}{|l|}{ \#35 mouth soreness } & \multirow[t]{4}{*}{$\mathrm{GxD} p=0.462 ;$ day $p=0.302$} \\
\hline Day 1 & $1.42(0.73)(n=43)$ & $1.33(0.69)(n=40)$ & $p=0.552$ & \\
\hline Day 5 & $1.26(0.72)(n=39)$ & $1.27(0.67)(n=33)$ & $p=0.921$ & \\
\hline Day paired $t$ test & $p=0.173(n=39)$ & $p=0.801(n=33)$ & & \\
\hline \multicolumn{4}{|c|}{ \#36 scores in corners of mouth } & \multirow[t]{4}{*}{$\mathrm{GxD} p=0.738 ;$ day $p=\mathbf{0 . 0 2 4}$} \\
\hline Day 1 & $1.28(0.59)(n=43)$ & $1.18(0.39)(n=40)$ & $p=0.348$ & \\
\hline Day 5 & $1.21(0.47)(n=39)$ & $1.06(0.24)(n=33)$ & $p=0.099 *$ & \\
\hline Day paired $t$ test & $p=0.160(n=39, d=0.23)$ & $p=\mathbf{0 . 0 4 4}(n=33, d=0.37)$ & & \\
\hline \multicolumn{4}{|l|}{ \#37 dry mouth } & \multirow[t]{4}{*}{$\mathrm{GxD} p=\mathbf{0 . 0 3 6}$; day $p<\mathbf{0 . 0 0 1}$} \\
\hline Day 1 & $3.12(0.80)(n=42)$ & $3.15(1.00)(n=40)$ & $p=0.878$ & \\
\hline Day 5 & $2.82(0.91)(n=39)$ & $2.39(1.00)(n=33)$ & $p=0.063(d=0.45)$ & \\
\hline Day paired $t$ test & $p=\mathbf{0 . 0 4 8}(n=38, d=0.33)$ & $p<\mathbf{0 . 0 0 1}(n=33 ; d=0.75)$ & & \\
\hline \multicolumn{4}{|l|}{ \#38 sticky saliva } & \multirow[t]{4}{*}{$\mathrm{GxD} p=0.347 ;$ day $p<\mathbf{0 . 0 0 1}$} \\
\hline Day 1 & $2.07(0.89)(n=42)$ & $2.23(0.99)(n=39)$ & $p=0.448$ & \\
\hline Day 5 & $1.76(0.82)(n=38)$ & $1.76(0.87)(n=33)$ & $p=0.978$ & \\
\hline Day paired $t$ test & $p=0.077(n=38, d=0.30)$ & $p=\mathbf{0 . 0 0 1}(n=33, d=0.64)$ & & \\
\hline \multicolumn{4}{|l|}{ \#39 food/drink sensitivity } & \multirow[t]{4}{*}{ GxD $p=0.490 ;$ day $p=0.080$} \\
\hline Day 1 & $2.10(1.09)(n=41)$ & $1.80(0.85)(n=40)$ & $p=0.176$ & \\
\hline Day 5 & $1.83(1.03)(n=36)$ & $1.64(0.86)(n=33)$ & $p=0.393$ & \\
\hline Day paired $t$ test & $p=0.091(n=34, d=0.30)$ & $p=0.500(n=33, d=0.12)$ & & \\
\hline \multicolumn{4}{|l|}{ \#40 taste different } & \multirow[t]{4}{*}{$\mathrm{GxD} p=0.760 ;$ day $p=\mathbf{0 . 0 2 1}$} \\
\hline Day 1 & $2.48(1.07)(n=42)$ & $2.05(0.93)(n=40)$ & $p=0.058$ & \\
\hline Day 5 & $2.24(1.15)(n=38)$ & $1.75(0.88)(n=32)$ & $p=\mathbf{0 . 0 4 9}(d=0.48)$ & \\
\hline Day paired $t$ test & $p=\mathbf{0 . 0 4 6}(n=37, d=0.34)$ & $p=0.206(n=32, d=0.23)$ & & \\
\hline \multicolumn{4}{|c|}{ \#41 problems eating solid food } & \multirow[t]{4}{*}{ GxD $p=0.689$; day $p=\mathbf{0 . 0 0 2}$} \\
\hline Day 1 & $1.65(1.02)(n=43)$ & $1.85(1.25)(n=40)$ & $p=0.429$ & \\
\hline Day 5 & $1.36(0.87)(n=39)$ & $1.52(0.94)(n=33)$ & $p=0.468$ & \\
\hline Day paired $t$ test & $p=\mathbf{0 . 0 0 8}(n=39, d=0.45)$ & $p=0.206(n=33, d=0.23)$ & & \\
\hline \multicolumn{4}{|c|}{ \#42 problems enjoying meals } & \multirow[t]{4}{*}{ GxD $p=0.401$, day $p=0.051$} \\
\hline Day 1 & $2.58(1.12)(n=43)$ & $2.28(1.19)(n=39)$ & $p=0.244$ & \\
\hline Day 5 & $2.49(1.25)(n=39)$ & $1.85(1.06)(n=33)$ & $p=\mathbf{0 . 0 2 2}(d=0.55)$ & \\
\hline Day paired $t$ test & $p=0.313(n=39, d=0.16)$ & $p=0.096(n=32, d=0.30)$ & & \\
\hline \multicolumn{5}{|c|}{ Patient perception of oral discomfort (0-10 NRS) } \\
\hline \multicolumn{4}{|l|}{ Mouth dryness } & \multirow[t]{7}{*}{$\mathrm{GxD} p=\mathbf{0 . 0 4 5}$; day $p<\mathbf{0 . 0 0 1}$} \\
\hline Day 1 & $5.4(2.3)(n=43)$ & $5.8(3.0)(n=41)$ & $p=0.560$ & \\
\hline Day 2 & $4.7(2.6)(n=41)$ & $4.0(3.1)(n=39)$ & $p=0.270$ & \\
\hline Day 3 & $3.8(2.6)(n=41)$ & $4.3(3.0)(n=38)$ & $p=0.440$ & \\
\hline Day 4 & $4.3(3.2)(n=39)$ & $4.0(3.0)(n=35)$ & $p=0.727$ & \\
\hline Day 5 & $4.6(2.7)(n=40)$ & $3.7(3.0)(n=33)$ & $p=0.187$ & \\
\hline Day $1-5$ paired $t$ test & $p=\mathbf{0 . 0 1 7}(n=40, d=0.39)$ & $p=\mathbf{0 . 0 0 1}(n=33, d=0.63)$ & & \\
\hline
\end{tabular}


Table 2 (continued)

\begin{tabular}{lllll}
\hline & $\begin{array}{l}\text { Control } \\
(n=44)\end{array}$ & $\begin{array}{l}\text { Intervention } \\
(n=44)\end{array}$ & $\begin{array}{l}p \text { value }(d \text { value }) \\
\text { for group } t \text { test }\end{array}$ & $\begin{array}{l}p \text { values for mixed } \\
\text { model effects }\end{array}$ \\
\hline Difficulty swallowing & & & \\
Day 1 & $1.4(2.4)(n=42)$ & $2.2(2.3)(n=41)$ & $p=0.144$ \\
Day 2 & $1.0(1.7)(n=41)$ & $1.6(2.4)(n=39)$ & $p=0.283^{*}$ \\
Day 3 & $0.7(1.5)(n=41)$ & $1.3(2.0)(n=38)$ & $p=0.170^{*}$ \\
Day 4 & $0.9(1.6)(n=39)$ & $1.5(2.1)(n=35)$ & $p=0.150$ \\
Day 5 & $0.9(1.5)(n=40)$ & $1.6(2.6)(n=33)$ & $p=0.183$ \\
Day 1-5 paired $t$ test & $p=0.091(n=39, d=0.28)$ & $p=0.549(n=33, d=0.11)$ & & \\
\hline
\end{tabular}

$G x D$ group by day interaction effect. $p$ values $<0.05$ are bolded. Cohen's $d$ is reported for within group $t$ tests when $p<0.10$ for either treatment group and is reported for day 5 between-group comparisons when $p<0.05$

* Separate variance $t$ test was used due to unequal variances

In our study sample, the most prevalent patient-reported oral symptom at baseline was dry mouth, with $78 \%$ reporting significant oral dryness on the EORTC QLQ-OH17 and 80\% reporting moderate to severe dry mouth on the NRS. This result is consistent with other studies in similar populations, where dry mouth has been reported in 77-78\% of terminally ill cancer patients $[1,8,36,37]$, with medication being the major cause [15]. In this study, patients were taking an average of 11 medications and $85 \%$ used opiates. The only group difference at baseline was in the use of opiates, $77 \%$ in the NS group and $93 \%$ in the $\mathrm{SO}$ group $(p=0.049)$. Despite this difference in use of opiates, no difference was found regarding presence of xerostomia at baseline. Anti-cancer treatment, such as chemotherapy, may cause xerostomia $[4,5]$, and $33 \%$ of the patients in this study were currently on palliative chemotherapy. However, there were no group differences at baseline with respect to type of chemotherapy or degree of xerostomia.

Although there was no significant difference in patientreported symptom scores in xerostomia between the groups after 4 days of intervention, there was a significant improvement over time within both groups. In addition, the effect size of the reduction in dry mouth over time was in the medium-tolarge range for the SO group and in the small-to-medium range for the NS group. Both rinses used in the present study had a positive effect on patients' subjective experience of xerostomia and could therefore be recommended for palliation of oral discomfort.

Dysgeusia, or taste alteration, is a frequently reported oral symptom in palliative care patients, with a prevalence of 60 $80 \%$ [38]. The prevalence of taste alteration among the patients in this study was similar to prior estimates, with more than $70 \%$ reporting that food/drinks tasted at least "a little" different (Fig. 2). Taste alteration may also impact patients' daily quality of life, food intake, and dysphagia, as well as be a symptom of oral fungal infection $[38,39]$. Given its prevalence and potential impact on other areas of function, taste alteration should be included in any assessment of oral symptoms.
Fig. 3 Changes in EORTC QLQ$\mathrm{OH} 17$ and NRS ratings based on dry mouth by treatment group (group-by-time interaction $p=0.036$ and 0.045 , respectively). For the EORTC QLQ-OH17, the effect size for the difference between groups on day 5 is Cohen's $d=0.45$; the effect size for improvement over time was $d=0.33$ for the saline group and 0.75 for the salvia group. For the NRS, the effect size for the difference between groups on day 5 is Cohen's $d=0.31$; the effect size for improvement over time was $d=0.39$ for the saline group, and 0.63 for the salvia group

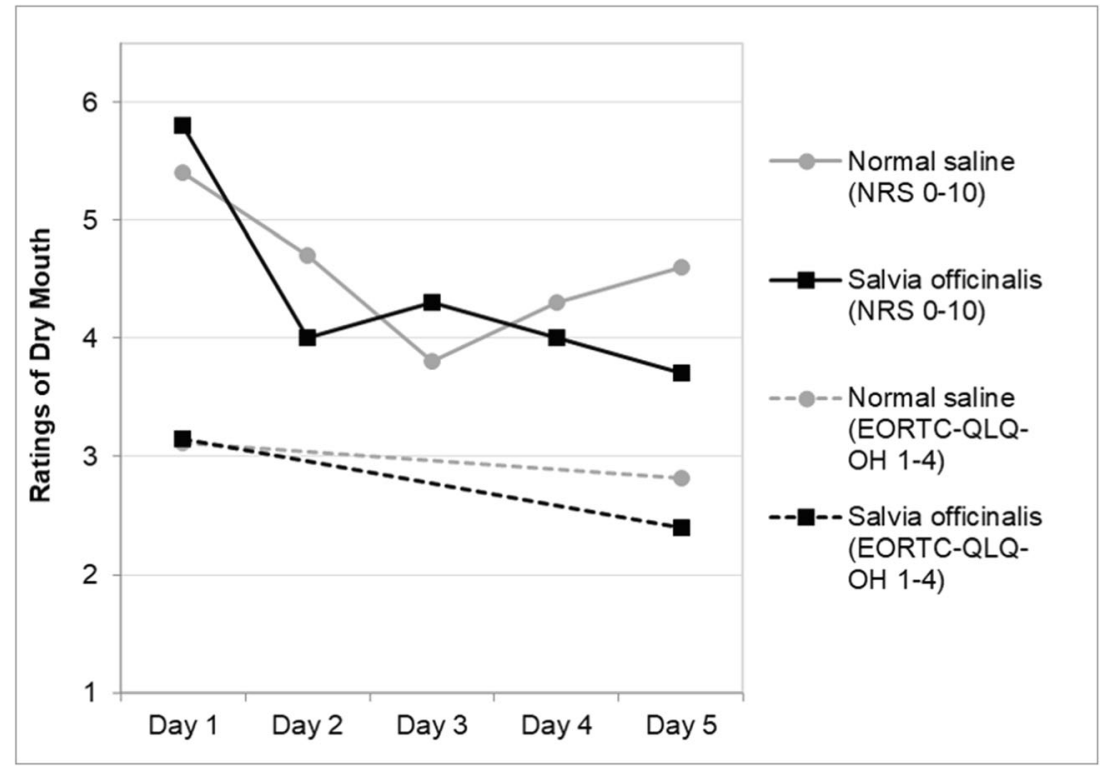


Table 3 Change in OMAS and clinical evaluation between days 1 and 5 by treatment group

\begin{tabular}{|c|c|c|c|c|}
\hline & $\begin{array}{l}\text { Control } \\
(n=44)\end{array}$ & $\begin{array}{l}\text { Intervention } \\
(n=44)\end{array}$ & $\begin{array}{l}p \text { value for } \\
\text { group } t \text { test }\end{array}$ & $\begin{array}{l}p \text { values for mixed } \\
\text { model effects }\end{array}$ \\
\hline \multicolumn{5}{|l|}{ OMAS } \\
\hline Ulceration score $(0-3)$ & & & & GxD $p=0.706 ;$ day $p=0.378$ \\
\hline Day 1 & $0.12(0.21)(n=44)$ & $0.13(0.29)(n=42)$ & $p=0.837$ & \\
\hline Day 5 & $0.09(0.17)(n=40)$ & $0.10(0.23)(n=33)$ & $p=0.750$ & \\
\hline Day paired $t$ test & $p=0.083(n=40, d=0.28)$ & $p=0.800(n=33, d=-0.04)$ & & \\
\hline Erythema score $(0-2)$ & & & & $\mathrm{GxD} p=0.481 ;$ day $p=\mathbf{0 . 0 2 2}$ \\
\hline Day 1 & $0.40(0.39)(n=44)$ & $0.34(0.30)(n=42)$ & $p=0.491$ & \\
\hline Day 5 & $0.31(0.35)(n=40)$ & $0.27(0.24)(n=33)$ & $p=0.506$ & \\
\hline Day paired $t$ test & $p=\mathbf{0 . 0 2 2}(n=40, d=0.38)$ & $p=0.498(n=33, d=0.12)$ & & \\
\hline \multicolumn{5}{|l|}{ Clinical evaluation } \\
\hline Plaque on tongue $(1-4)$ & & & & $\mathrm{GxD} p=0.854 ;$ day $p=\mathbf{0 . 0 0 3}$ \\
\hline Day 1 & $2.02(0.74)(n=43)$ & $2.07(1.11)(n=42)$ & $p=0.815$ & \\
\hline Day 5 & $1.70(0.79)(n=40)$ & $1.76(0.75)(n=33)$ & $p=0.752$ & \\
\hline Day paired $t$ test & $p=\mathbf{0 . 0 1 8}(n=39, d=0.40)$ & $p=0.083(n=33, d=0.31)$ & & \\
\hline Plaque on teeth $(1-4)$ & & & & $\mathrm{GxD} p=0.279 ;$ day $p<\mathbf{0 . 0 0 1}$ \\
\hline Day 1 & $1.66(0.68)(n=44)$ & $2.00(0.95)(n=41)$ & $p=0.059$ & \\
\hline Day 5 & $1.45(0.60)(n=40)$ & $1.61(0.61)(n=33)$ & $p=0.274$ & \\
\hline Day paired $t$ test & $p=\mathbf{0 . 0 1 8}(n=40, d=0.39)$ & $p=\mathbf{0 . 0 0 8}(n=33, d=0.49)$ & & \\
\hline Sign of oral dryness & & & & GxD $p=0.385 ;$ day $p=\mathbf{0 . 0 0 1}$ \\
\hline Day 1 & $45 \%(18 / 40)$ & $49 \%(19 / 39)$ & $p=0.741$ & \\
\hline Day 5 & $20 \%(7 / 35)$ & $32 \%(10 / 31)$ & $p=0.256$ & \\
\hline Day McNemar test & $p=\mathbf{0 . 0 2 2}(n=35)$ & $p=0.125(n=30)$ & & \\
\hline
\end{tabular}

$G x D$ group by day interaction effect. $p$ values $<0.05$ are bolded. Cohen's $d$ is reported for within group $t$ tests when $p<0.10$ for either treatment group

At baseline, $90 \%$ of patients reported brushing their teeth twice daily or more. Despite good oral hygiene and dental status, clinical evaluation indicated that $76 \%$ had evidence of mild erythema. In a prior study with terminally ill cancer patients, 50\% had erythema assessed by the OMAS [7]. Although patients on or in need of antifungal treatment based on clinical signs were excluded from our study, oral fungal infection among cancer patients in palliative care may occur with either asymptomatic and symptomatic features [40]. Erythema reported in the current study could thus be related to ongoing or previous chemotherapy, oral dryness, and/or an asymptomatic oral fungal infection. At baseline, none of the patients was being treated with antifungal medication nor did they show obvious clinical signs of oral candidiasis. However, a swab test for Candida carriage was taken at baseline of all patients and further fungal analyses will be presented in another study.

This study followed the procedures published at ClinicalTrials.gov, but has several limitations. Most importantly, the observed improvement in the two groups is likely not due to the mouth rinse alone, but is also likely the result of the structured mouth care regimen, of which regular rinsing was only part. Moreover, most of the patients were also using lubricant products, even at baseline. Given that $80 \%$ of patients with advanced cancer report dry mouth and these products are routinely used in the treatment of dry mouth, it was considered unethical to request that patients stop using them. Because some of the participants were in need of oral lubricant, the oral care protocol was standardized to include use of oral gel and lip balm in order to minimize group differences. Additionally, due to SO's distinctive taste and smell, patients were not blinded to their group assignment.

Although patients did not compare the SO and NS rinses with each other, both rinses received ratings indicating that patients were generally satisfied with the one they received. Both SO and NS are inexpensive rinses, and being alcoholfree, they are likely to be gentle to the mucosa in the oral cavity. However, the SO mouth rinse is also drinkable, and therefore harmless for patients with swallowing disorders and problems with spitting out the mouth rinse.

Clinical studies in palliative care must utilize procedures that are as minimally burdensome as possible to the patients. Questionnaires must be brief and easy to answer and clinical examinations must be kept as simple as possible [41]. This was the reason for excluding items from the EORTC QLQ- 
OH17 questionnaire, as some items (\#43-\#47) were considered less applicable to our study group given the patients' advanced disease and situation. The study procedure was designed with consideration of these challenges, but future studies in this area would be strengthened by addressing these limitations. Lastly, it is worth noting that only 22 patients (14\%) declined to participate, although an additional 24 patients initially agreed, but later declined. This shows that patients at end-of-life often want to contribute to clinical trials despite their fragile situation.

\section{Conclusion}

The results of this study indicate that the Salvia officinalis mouth rinse intervention was as good as, but not significantly better than normal saline rinsing for reducing oral symptoms, particularly dry mouth among cancer patients in palliative care. Rinsing four times a day with either solution, together with a basic oral care program, improved patients' oral status and reduced oral discomfort. It would be a considerable improvement for palliative care units to collaborate with and include oral health professionals to identify and manage oral health issues in order to achieve the best patient care and quality of life for these patients.

Acknowledgements The authors are grateful to the staff at the inpatient unit at Hospice Lovisenberg, Lovisenberg Diaconal Hospital for their contributions and support with the study procedures so the patients could participate and complete the study. A special thanks to Oddveig Tallang for helping to recruit patients and to Marianne Dahl for collecting data. We also like to thank the Bundi Cancer Support Foundation, and Kirsten Rønnings Endowment, for supporting the study.

Author contribution AT, BBH, AL, TS, ES, and KGF designed the trial and AT drafted the protocol. Material preparation, data collection, and analysis were performed by BBH, REM, ES CG, KGF, LHH, JS, TS, and AL. CG conducted the statistical analyses and assisted in the preparation of the manuscript. KEM contributed his expertise on natural products/ pharmacognosy about Salvia officinalis and assisted in the preparation of the manuscript. AL, BBH, CG, KGF, LHH, and REM contributed to the interpretation of the results and preparation of the manuscript. The first draft of the manuscript was written by REM and CG and all authors provided input on versions of the manuscript and approved the final manuscript.

Funding Open access funding provided by University of Oslo (incl Oslo University Hospital). This study was funded by Lovisenberg Diaconal Hospital, Oslo, the Bundi Cancer Support Foundation, Oslo, Kirsten Rønnings Endowment, Oslo, and South-Eastern Norway Regional Health Authority (reference \#2021026), all in Norway.

\section{Declarations}

Ethics approval and consent to participate All study procedures involving human participants were in accordance with the 1964 Helsinki Declaration and its later amendments or comparable ethical standards, and with the ethical standards of the Regional Committee for Medical Research Ethics, Health Region South-East Norway, which approved the study in October 2013 (reference \#2013/1531). Informed consent was obtained from all participants included in the study.

Consent for publication Patients have not signed informed consent regarding publishing their data.

Competing interests The authors declare no competing interests.

Open Access This article is licensed under a Creative Commons Attribution 4.0 International License, which permits use, sharing, adaptation, distribution and reproduction in any medium or format, as long as you give appropriate credit to the original author(s) and the source, provide a link to the Creative Commons licence, and indicate if changes were made. The images or other third party material in this article are included in the article's Creative Commons licence, unless indicated otherwise in a credit line to the material. If material is not included in the article's Creative Commons licence and your intended use is not permitted by statutory regulation or exceeds the permitted use, you will need to obtain permission directly from the copyright holder. To view a copy of this licence, visit http://creativecommons.org/licenses/by/4.0/.

\section{References}

1. Wilberg P, Hjermstad MJ, Ottesen S, Herlofson BB (2012) Oral health is an important issue in end-of-life cancer care. Support Care Cancer 20:3115-3122. https://doi.org/10.1007/s00520-012-1441-8

2. Venkatasalu MR, Murang ZR, Ramasamy DTR, Dhaliwal JS (2020) Oral health problems among palliative and terminally ill patients: an integrated systematic review. BMC Oral Health 20: 79. https://doi.org/10.1186/s12903-020-01075-w

3. Krishnasamy M (1995) Oral problems in advanced cancer. Eur J Cancer Care 4:173-177

4. Jensen SB, Pedersen AML, Vissink A, Andersen E, Brown CG, Davies AN, Dutilh J, Fulton JS, Jankovic L, Lopes NN (2010) A systematic review of salivary gland hypofunction and xerostomia induced by cancer therapies: prevalence, severity and impact on quality of life. Support Care Cancer 18:1039-1060. https://doi. org/10.1007/s00520-010-0827-8

5. Wilberg P, Hjermstad MJ, Ottesen S, Herlofson BB (2014) Chemotherapy-associated oral sequelae in patients with cancers outside the head and neck region. J Pain Symptom Manag 48: 1060-1069. https://doi.org/10.1016/j.jpainsymman.2014.02.009

6. Norwegian Directorate of Health (10/2019) The Norwegian guidelines for palliative care in cancer care (IS - 2800). Norwegian Directorate of Health, Oslo, Norway. https:/www.helsedirektoratet. no/retningslinjer/palliasjon-i-kreftomsorgen-handlingsprogram

7. Fischer D, Epstein J, Yao Y, Wilkie D (2014) Oral health conditions affect functional and social activities of terminally ill cancer patients. Support Care Cancer 22:803-810

8. Hanchanale S, Adkinson L, Daniel S, Fleming M, Oxberry SG (2015) Systematic literature review: xerostomia in advanced cancer patients. Support Care Cancer 23:881-888

9. Epstein JB, Beier Jensen S (2015) Management of hyposalivation and xerostomia: criteria for treatment strategies. Compend Contin Educ Dent 36:600-603

10. Mercadante S, Aielli F, Adile C, Ferrera P, Valle A, Fusco F, Caruselli A, Cartoni C, Massimo P, Masedu F (2015) Prevalence of oral mucositis, dry mouth, and dysphagia in advanced cancer patients. Support Care Cancer 23:3249-3255

11. Sweeney MP, Bagg J, Baxter WP, Aitchison TC (1997) Clinical trial of a mucin-containing oral spray for treatment of xerostomia in hospice patients. Palliat Med 11:225-232 
12. Davies AN, Daniels C, Pugh R, Sharma K (1998) A comparison of artificial saliva and pilocarpine in the management of xerostomia in patients with advanced cancer. Palliat Med 12:105-111

13. Davies AN (2000) A comparison of artificial saliva and chewing gum in the management of xerostomia in patients with advanced cancer. Palliat Med 14:197-203

14. Furness S, Worthington HV, Bryan G, Birchenough S, McMillan R (2011) Interventions for the management of dry mouth: topical therapies. Cochrane Database Syst Rev:CD008934. https://doi. org/10.1002/14651858.CD008934.pub2

15. De Conno F, Martini C, Sbanotto A, Ripamonti C, Ventafridda V (2011) Mouth care. In: Hanks G, Cherny NI, Christakis NA, Fallon M, Kaasa S, Portenoy RK (eds) Oxford Textbook of Palliative Medicine, Fourth edn. Oxford University Press, New York, pp 996-1011

16. Milligan S, McGill M, Sweeney MP, Malarkey C (2001) Oral care for people with advanced cancer: an evidence-based protocol. Int $\mathrm{J}$ Palliat Nurs 7:418-426

17. Kvalheim SF, Marthinussen MC, Haugen DF, Berg E, Strand GV, Lie SA (2019) Randomized controlled trial of the effectiveness of three different oral moisturizers in palliative care patients. Eur $\mathrm{J}$ Oral Sci 127:523-530. https://doi.org/10.1111/eos.12655

18. Farah CS, McIntosh L, McCullough MJ (2009) Mouthwashes. https://www.nps.org.au/australian-prescriber/articles/ mouthwashes. Accessed March 2020

19. Hubbert M, Sievers H, Lehnfeld R, Kehrl W (2006) Efficacy and tolerability of a spray with Salvia officinalis in the treatment of acute pharyngitis-a randomised, double-blind, placebo-controlled study with adaptive design and interim analysis. Eur J Med Res 11:20-26

20. Lalicevic S (2004) Comparison of benzydamine hydrochloride and Salvia officinalis as an adjuvant local treatment to systemic nonsteroidal anti-inflammatory drug in controlling pain after tonsillectomy, adenoidectomy, or both: an open-label, single-blind, randomized clinical trial. Curr Ther Res Clin Exp 65:360-372

21. Barnes J, Anderson LA, Phillipson JD (2007) Herbal medicines, 3rd edn. Pharmaceutical Press, London

22. Ghorbani A, Esmaeilizadeh M (2017) Pharmacological properties of Salvia officinalis and its components. J Tradit Complement Med 7:433-440

23. Fossum G, Malterud K, Østensvig A (2016) Assessment report on Salvia officinalis L., folium and Salvia officinalis L., aetheroleum. European Medicines Agency. https://www.ema.europa.eu/en/ documents/herbal-report/final-assessment-report-salvia-officinalis1-folium-salvia-officinalis-1-aetheroleum-revision-1 en.pdf Accessed 29 March 2020

24. De Oliveira JR, Vilela P, Almeida RA, De Oliveira FE, Carvalho CAT, Camargo SEA, Jorge AOC, de Oliveira LD (2019) Antimicrobial activity of noncytotoxic concentrations of Salvia officinalis extract against bacterial and fungal species from the oral cavity. Gen Dent 67:22-26

25. Oral Health in Patients with Advanced Cancer. https:// lovisenbergsykehus.no/fag-og-forskning/forskningsprosjekter/ doktorgradsprosjekter. Accessed April 2020

26. Committee on Herbal Medicinal Products (2009) Community Herbal Monograph on Salvia officinalis L. Folium, London
27. Schag CC, Heinrich RL, Ganz P (1984) Karnofsky performance status revisited: reliability, validity, and guidelines. J Clin Oncol 2:187-193

28. Hjermstad MJ, Bergenmar M, Fisher SE, Montel S, NicolatouGalitis O, Raber-Durlacher J, Singer S, Verdonck-de Leeuw I, Weis J, Yarom N (2012) The EORTC QLQ-OH17: a supplementary module to the EORTC QLQ-C30 for assessment of oral health and quality of life in cancer patients. Eur J Cancer 48:2203-2211

29. Aaronson NK, Ahmedzai S, Bergman B, Bullinger M, Cull A, Duez NJ, Filiberti A, Flechtner H, Fleishman SB, de Haes JCJM, Kaasa S, Klee M, Osoba D, Razavi D, Rofe PB, Schraub S, Sneeuw K, Sullivan M, Takeda F (1993) The European Organization for Research and Treatment of Cancer QLQ-C30: a quality-of-life instrument for use in international clinical trials in oncology. J Natl Cancer Inst 85:365-376

30. Hjermstad MJ, Bergenmar M, Bjordal K, Fisher SE, Hofmeister D, Montel S, Nicolatou-Galitis O, Pinto M, Raber-Durlacher J, Singer $S$ (2016) International field testing of the psychometric properties of an EORTC quality of life module for oral health: the EORTC QLQOH15. Support Care Cancer 24:3915-3924

31. Henricsson V, Svensson A, Olsson H, Axéll T (1990) Evaluation of a new device for measuring oral mucosal surface friction. Eur J Oral Sci 98:529-536

32. Henriksen BM, Ambjørnsen E, Axéll TE (1999) Evaluation of a mucosal-plaque index (MPS) designed to assess oral care in groups of elderly. Spec Care Dentist 19:154-157. https://doi.org/10.1111/j. 1754-4505.1999.tb01378.x

33. Sonis ST, Eilers JP, Epstein JB, LeVeque FG, Liggett WH, Mulagha MT, Peterson DE, Rose AH, Schubert MM, Spijkervet FK (1999) Validation of a new scoring system for the assessment of clinical trial research of oral mucositis induced by radiation or chemotherapy. Cancer 85:2103-2113

34. Cohen J (1992) A power primer. Psychol Bull 112:155-159

35. Faul F, Erdfelder E, Lang A-G, Buchner A (2007) G* Power 3: a flexible statistical power analysis program for the social, behavioral, and biomedical sciences. Behav Res Methods 39:175-191

36. Sweeney MP, Bagg J (2000) The mouth and palliative care. Am J Hosp Palliat Care 17:118-124

37. Fleming M, Craigs CL, Bennett MI (2020) Palliative care assessment of dry mouth: what matters most to patients with advanced disease? Support Care Cancer 28:1121-1129. https://doi.org/10. 1007/s00520-019-04908-9

38. Heckel M, Stiel S, Ostgathe C (2015) Smell and taste in palliative care: a systematic analysis of literature. Eur Arch Otorhinolaryngol 272:279-288

39. Hovan AJ, Williams PM, Stevenson-Moore P, Wahlin YB, Ohrn KE, Elting LS, Spijkervet FK, Brennan MT (2010) A systematic review of dysgeusia induced by cancer therapies. Support Care Cancer 18:1081-1087

40. Davies AN, Brailsford SR, Beighton D (2006) Oral candidosis in patients with advanced cancer. Oral Oncol 42:698-702

41. Cooke C, Ahmedzai S, Mayberry J (1996) Xerostomia-a review. Palliat Med 10:284-292

Publisher's note Springer Nature remains neutral with regard to jurisdictional claims in published maps and institutional affiliations. 